\title{
On the relation between stimulus intensity and processing time: Piéron's law and choice reaction time
}

\author{
DELPHINE PINS and CLAUDE BONNET \\ Université Louis Pasteur, Strasbourg, France
}

\begin{abstract}
Piéron $(1914,1920,1952)$ demonstrated that simple reaction time (SRT) decays as a hyperbolic function of luminance in detection tasks. However, whether such a relationship holds equally for choice reaction time (CRT) has been questioned (Luce, 1986; Nissen, 1977), at least when the task is not brightness discrimination. In two SRT and three CRT experiments, we investigated the function that relates reaction time (RT) to stimulus intensity for five levels of luminance covering the entire mesopic range. The psychophysical experiments consisted of simple detection, two-alternative forced choice ( $2 \mathrm{AFC}$ ) with spatial uncertainty, $2 \mathrm{AFC}$ with semantic categorization, and $2 \mathrm{AFC}$ with orientation discrimination. The results of the experiments showed that mean RT increases with task complexity. However, the exponents of the functions relating RT to stimulus intensity were found to be similar in the different experiments. This finding indicates that Piéron's law holds for CRT as well as for SRT. It describes RT as a power function of stimulus intensity, with similar exponents, regardless of the complexity of the task.
\end{abstract}

It has been known since the last century that simple reaction time (SRT) decreases when the intensity of a given stimulus increases (e.g., Cattell, 1886; Exner, 1868; Piéron, 1914, 1920; Wundt, 1886). Piéron described this effect by the following power function (Piéron, 1914, $1920,1952)$ :

$$
(\mathrm{SRT})-t_{0}=\beta I^{-\alpha}
$$

where SRT is simple reaction time, $t_{0}$ an asymptotic RT reached at higher stimulus intensities, $\beta$ the range of changes between the $t_{0}$ value and the maximum RT determined at threshold, $I$ the intensity of the stimulus, and $\alpha$ the exponent of the function. The parameters $\alpha$ and $t_{0}$ appear to be specific for a given sensory modality (see Bonnet, 1992a, 1992b), and $t_{0}$ appears to represent the combination of two constant parameters: the duration of the motor component and a specific processing time for a given sensory modality. Since $t_{0}$ varies between sensory modalities, its functional significance is presumably more sensory than decisional (see Bonnet, 1992b). However, its estimation is difficult (see Luce, 1986) and depends very much on the range and/or the number of intensities. When the range of intensities does not extend high enough, it is safer to reduce Piéron's function to two

We should like to thank S. Link and B. Dresp for their helpful discussions and comments on a previous version of the manuscript. Critical comments by D. R. J. Laming and an anonymous referee have helped us a lot, and we thank them both. Correspondence should be addressed to D. Pins, Université Louis Pasteur, Laboratoire de Psychophysique Sensorielle, LN2C URA1939 C.N.R.S., 12 rue Goethe, F-67000 Strasbourg, France (e-mail:psyphys@isis.u-strasbg.fr). parameters $(\alpha, \beta)$, although the generality of the exponent cannot be guaranteed.

Other functions have been found to account for the relation between RT and stimulus intensity such as, for instance, Michaelis's function, ${ }^{1}$ which describes the responses of the visual receptor (Easland \& Wasserman, 1979; Hemilä, 1987; Naka \& Rushton, 1966). Hinton and Sejnowski (1986) proposed a logistic function to describe these phenomena, but this does not appear to account for the empirical data generally (see, e.g., Schweickert, Dahn, \& McGuigan, 1988). Our findings suggest that Michaelis's and Piéron's functions may fit SRT and choice reaction time CRT equally well, so that the choice of Piéron's function, as in the present study, is not critical with respect to our aim.

Although such an effect of stimulus intensity on RT has been questioned for CRT tasks when visual stimuli are used (see, e.g., Luce, 1986), some authors have shown that CRT decreases when the luminance of the stimulus increases (e.g., Lappin \& Disch, 1972; Pachella \& Fisher, 1969; Posner, 1986). One main goal in this paper is to demonstrate that there is an effect of intensity on CRT and that Piéron's function applies equally well to SRT and CRT tasks using similar stimuli.

Intensity is not the only factor that produces changes in RT. How the effect of intensity on RT combines with effects of other experimental factors such as different levels of task complexity is, in CRT, still an open question. Some authors have previously mentioned examples for an additivity of the effects of intensity and other factors (e.g., Schweickert et al., 1988). The additivity may be explained in terms of serial processing of stimulus intensity and other dimensions of the task. It can be assumed 
that the processing of other dimensions can begin only when intensity processing has come to an end (however, see Miller, 1993). It is then postulated that, at a very early level of neural processing, the first available information concerns the intensity per se of the visual stimulus, irrespective of further coding such as that concerning localization, orientation, shape, and so on. This first step will thus be referred to here simply as "intensity processing." Consequently, it then would be appropriate to determine whether such a processing time combines additively with task complexity in CRT, as explained below.

Most SRT models assume that responses result basically from some kind of accumulation of information in time (see, e.g., Grice, 1968; Laming, 1968; Link, 1978, 1992; Luce, 1986; Luce \& Green, 1972; McGill, 1961, $1963)$. When intensity increases, the rate of neural impulses increases (see, e.g., Bartlett \& Doty, 1974; Boynton \& Whitten, 1970; Levick, 1973; Marrocco, 1975; Stone \& Fukuda, 1974; Tepas \& Armington, 1962). Therefore, the accumulation of the relevant information, which is generally called "accrual," can be expected to be faster. In an SRT task, a response is assumed to be initiated when a critical level of accumulation is reached. ${ }^{2}$ Consequently, the relationship between SRT and stimulus intensity should reveal the relative duration of the accumulation process (see, e.g., Schweickert et al., 1988).

In fact, most SRT models assume that a given RT depends on at least two components: the rate of accrual and the critical accumulation level at which the response is triggered. Therefore, in a given experiment with several levels of luminance, the psychophysical relationship between RT and intensity (as described by Piéron's function) may reflect a change in the rate of accrual, provided that the critical level is constant. Consequently, these models assume that the rate of accrual depends only on stimulus intensity while the critical level of accumulation is under the subject's control. As such, it is a priori independent from the rate of accrual. Most of the additivity effects mentioned in the literature concern factors known to affect the decisional process (i.e., the one that is under the subject's control). These factors are foreperiod duration, similarity, stimulus-response compatibility, practice, or number of response alternatives (e.g., Biederman \& Kaplan, 1970; Everett, Hochhaus, \& Brown, 1985; Schweickert et al., 1988).

Accrual models are directly relevant to SRT tasks, which are basically detection tasks, and, as such, SRT mainly reflects the processing time for a given stimulus intensity. The question here, as raised previously by Nissen (1977), is to find out what happens when the task involves more cognitive processes than just the processing of intensity, as in the case of CRT tasks.

A similar early processing of intensity can be assumed for both SRT and CRT tasks. However, in the latter case, the critical level of accumulation refers to the beginning of the discrimination process within an additive model (see note 2 ). Increasing luminance can therefore be ex- pected to reduce CRT as well as SRT. Now, if in a given situation a choice must be made between two modalities of one dimension (for instance, orientation discrimination) that is, in theory, separable from the luminance lev-' els (see, e.g., Garner, 1974), this choice can be expected to add a constant time to the intensity processing time. Such a result can be obtained only if, on the one hand, the response criterion ${ }^{3}$ is independent of stimulus intensity, and on the other hand, the critical level of accumulation is independent of the subsequent discrimination process. This critical level of accumulation would then be the same in SRT and CRT tasks.

Hence, one may expect that Piéron's function will be parallel to the one obtained in SRT tasks, with an equal exponent. On a more general level, it may be expected that the exponent of Piéron's function will not change, whatever the complexity of the additional stages required between luminance processing and the decision process. This implies that within a given experimental condition, a change in RT as a function of luminance would arise only as a consequence of the first level of processing.

Five experiments, with identical luminance levels, were designed in order to shed light on these questions. These experiments were of increasing complexity with respect to the involved levels of processing. First, two SRT tasks were run with spatial uncertainty in order to keep the same stimuli as those used in CRT tasks. These two experiments enabled us to collect basic data for estimates of the exponent of Piéron's function. Then, two choice tasks, with different complexity levels, but both with a high stimulus-response compatibility, followed. One consisted of two-alternative forced choice ( $2 \mathrm{AFC}$ ) with spatial uncertainty, and the other task required 2 AFC with semantic categorization. The present spatial discrimination tasks (at different luminance levels) did not involve a discrimination between luminance levels.

However, complexity may be multidimensional. It may lead to a change in the level of perceptual information processing, and it may also engender a change in the decision process as, for instance, when there are changes in stimulus-response compatibility. As mentioned above, the absence of stimulus-response compatibility is known to have an influence on the decision process (Biederman \& Kaplan, 1970) and, consequently, to affect the response criterion. Therefore, an additional task was run in which there was no stimulus-response compatibility; this was a 2 AFC orientation discrimination.

When there is no stimulus-response compatibility, the response criterion position can be expected to be higher than in the case of compatibility. With practice, as preliminary experiments seemed to show, the effect of the no stimulus-response compatibility on the decision process decreases. This can be explained by a decrease of the response criterion. When the response becomes automatic, it may be expected that the criterion will be as low as it is in the other experiments. Consequently, with well-trained subjects, Piéron's law should apply in the same way as in the other experiments. 


\section{PRELIMINARY EXPERIMENT}

Previous experiments, by Piéron (1914, 1920); Vaughan, Costa, and Gilden (1966); and Mansfield (1973), validated Piéron's function in vision for the relationship between SRT and luminance. These authors used a very large range of luminance from the low scotopic to the high photopic levels. An exponent of $-\alpha=-0.33$ fits their results nicely. The first aim of the present experiment was to verify that the equipment we intended to use (a video monitor) would lead to similar results. A secondary aim of the experiment was to provide relevant reference data for the design of further experiments, as explained in the discussion.

\section{Method}

Subject. One trained subject, the first author, took part in the experiment. She had corrected-to-normal vision.

Procedure. The stimuli were presented binocularly on a highresolution video monitor (Visionor Model M 5l CHR No. 1007 , Lille, France). They were generated through a PC compatible computer (HP20 80386) using a special graphics adaptor (GALAXY ref. SA-1019A, Evroz, Tel Aviv) providing a display of $1,024 \times$ 768 pixels at $60-\mathrm{Hz}$ frame rate (noninterlaced). Careful calibration of each R-G-B combination was carried out with a CS 100 Minolta photometer and cross-checked with another device standardized to a Pritchard photomultiplier.

The stimuli (Figure 1) were luminous rectangles $\left(30 \times 7.5^{\prime}\right.$ of arc) shown on a dark background $\left(0.02 \mathrm{~cd} / \mathrm{m}^{2}\right)$ in a dark room. The subject was placed at a viewing distance of $70 \mathrm{~cm}$. The head position was stabilized by means of a chin rest. She was looking at a central fixation dot (1.5 of are diam., $\left.10 \mathrm{~cd} / \mathrm{m}^{2}\right)$, and in each trial, a luminous rectangle appeared with its center located at $1.25^{\circ}$ to the left or to the right of the fixation point.

The luminance levels ranged from threshold to photopic levels. The different intensities were presented in different experimental blocks of trials in order to avoid adaptation effects due to changes of luminance from trial to trial. Since the expected intensity function is a hyperbolic curve, these levels of luminance follow an approximately geometrical series. Each of 15 luminance levels $(0.16$, $0.18,0.22,0.25,0.28,0.45,0.81,1.41,3.08,5.54,10.01,17.94$, 32.30 , and $58.18 \mathrm{~cd} / \mathrm{m}^{2}$ ) were presented in five experimental sessions consisting of pure blocks (using one luminance level) of 100 trials. Thus 500 RTs were recorded for each luminance level. The median RT was calculated for each session and the medians were averaged over sessions.

Presentation time was about $83 \mathrm{msec}$ (five frames). RT measurements were rounded to the closest millisecond as indicated by the external clock driven by the computer. The appearance of the

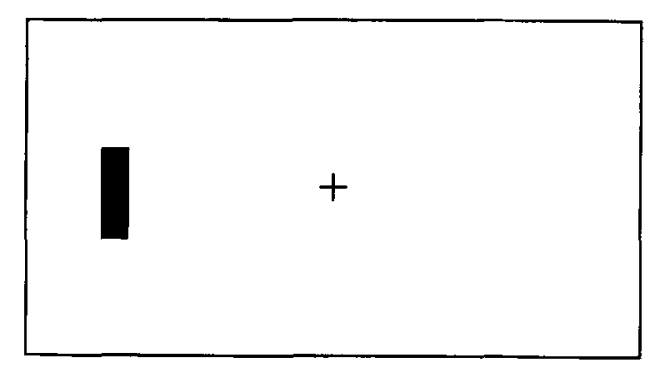

Figure 1. One of the stimuli presented in the preliminary experiment and in Experiments 1 and 3, with opposite contrast. A luminous rectangle appeared at $1.25^{\circ}$ left of fixation.

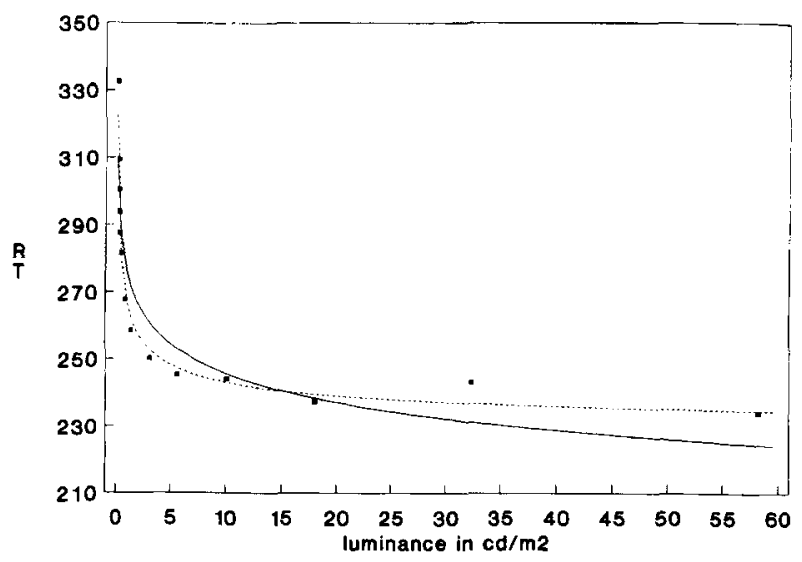

Figure 2. The curves show Piéron's function fitted to the mean data of the preliminary experiment. The solid line is the two-parameter Piéron's function and the dotted line the three-parameter Piéron's function. The squares (above and below the curves) are the observed mean reaction time (RT) medians.

target was preceded by an auditory warning signal $(1000 \mathrm{~Hz}$, $500 \mathrm{msec})$. Five preparatory periods $(450,550,650,750$, and $850 \mathrm{msec}$ ) were used and presented randomly according to an exponential distribution in order to minimize anticipatory responses. At the beginning of each session, the subject was dark adapted, and pauses were made between blocks of different luminances.

The previous authors used SRT tasks. We chose to use here a CRT task, in which the subject had to decide whether the stimulus appeared to the left (left key) or to the right (right key) of the fixation point (high spatial compatibility).

\section{Results}

As shown in Figure 2, CRT decreased with increasing luminance. First, the three parameters of the classical Piéron's function were estimated on the present results. An iterative procedure was used to estimate parameters. For successive values of $t_{0}$, the parameters $\alpha$ and $\beta$ were estimated using a least squares method on the logtransformed data. The procedure stopped when the best correlation was found. The best fit $(r=-.98)$ was obtained with (Figure 2):

$$
\begin{aligned}
-\alpha & =-0.3864, \\
t_{0} & =226 \mathrm{msec} .
\end{aligned}
$$

The exponent thus appears to be very close to the one previously reported. Furthermore, the luminance level at which the RT function reaches its asymptotic level $\left(t_{0}\right)$ is estimated to be $37 \mathrm{~cd} / \mathrm{m}^{2}$, a value that is very similar to the $40 \mathrm{~cd} / \mathrm{m}^{2}$ estimated by Mansfield (1973) on the basis of his results.

Second, for the sake of comparison with the results of further experiments, the two parameters $(-\alpha$ and $\beta$ ) were estimated for the same data leaving out the $t_{0}$ value (Figure 2). Although the goodness of the fit is slightly lower $(r=-.94)$, it is still highly acceptable. In such a case, the exponent of Pieron's function turns out to be as follows:

$$
-\alpha=-0.0513
$$




\section{Conclusion}

This preliminary experiment confirms the validity of the estimates of the parameters of the Piéron's function given by previous authors (Piéron, 1914, 1920; Vaughan et al., 1966; Mansfield, 1973). Second, it provides a first result indicating that simple and choice RTs can lead to similar estimates of the exponent. The mean CRT is, however, a little longer.

The different intensities were presented here in separate experimental blocks. With such a presentation, subjects may have difficulty in maintaining a constant response criterion from block to block and from intensity to intensity. In the next experiments, we decided to present a more restricted number of luminance levels randomly presented within blocks of trials (mixed blocks). The reason for using such mixed blocks was to ensure the stability of the criterion level within an experiment (Grice, 1968; Grice \& Hunter, 1964). Second, in order to reduce difficulties due to changes in adaptation levels when using different intensities from trial to trial, we decided to restrict the range of luminance levels to the mesopic range of luminances. In fact, according to Mansfield's (1973) results, the mesopic region is the one with the largest variations in the tangent slope of Piéron's function.

\section{Piéron's Law in SRT Tasks}

Two experiments were run in order to gather data for the estimation of the parameters of Piéron's function in SRT tasks with different stimuli for a given range of luminance levels.

\section{EXPERIMENT 1 SRT and Localization}

In this first experiment, we presented a detection task with spatial uncertainty. That uncertainty (left/right position of the stimulus) was introduced for the sake of consistency between the SRT task and the CRT task presented later. According to Piéron's function, SRT should decrease when the luminance of the stimulus increases.

\section{Method}

Subjects. Four trained subjects took part in the experiment. One of them was the first author. They all had normal or corrected-tonormal vision.

Procedure. Stimuli and experimental design were identical to those of the preliminary experiment. However, in order to ensure the constancy of the response criterion within an experimentai session, the luminance levels were presented in mixed blocks, as explained above. Five levels of luminance of the target, covering the entire mesopic range, were used: $0.28,0.45,0.81,1.41$, and $3.09 \mathrm{~cd} / \mathrm{m}^{2}$. These levels of luminance follow an approximately geometrical series, and were easily discriminable as confirmed by another preliminary experiment. The averaged Weber ratio for successive presentations of pairs of luminance was found to be $16.5 \%$ for that range of luminance. The difference between two adjacent levels of luminance was thus about 3 JNDs. The same five levels of luminance were used in all the following experiments of the present paper.

Within a given session, the two spatial locations (left/right) and the five luminance levels were randomly presented from trial to trial. The use of a parafoveal stimulus location was chosen for two reasons: First, the two lowest levels of luminance were barely visible foveally (low mesopic levels); second, the tasks in the following experiments were CRT tasks, in which the subject bad to choose whether a given characteristic of the stimulus was presented to the left or to the right of the fixation point. In this experiment, a single response key was used. A preliminary experiment with the same stimuli had shown that retinal eccentricity had no effect on Piéron's function. We chose a small eccentricity to facilitate the form identification task (see Experiment 4), and to create a condition in which the target could be detected at low mesopic levels of luminance.

The same presentation time as in the preliminary experiment was used $(83 \mathrm{msec})$. This presentation duration is beyond the limit of temporal summation of Bloch's law (see Graham, 1965; Mansfield, 1973; Ueno, 1977). Each experimental session contained 500 trials and the subjects were dark adapted. The task was to press a response key as soon as the stimulus appeared, regardless of its location.

Three subjects went through five experimental sessions, the other one (E.D.) only through four experimental sessions. Thus 500 or 400 RTs were recorded for each level of luminance. In all the following experiments, the median RT was calculated for each session and the medians were averaged over sessions.

\section{Results}

The mean difference between the median RT, according to the location (left/right) of thestimulus, was about $1 \mathrm{msec}$. The data were averaged over the localization factor.

In all the experiments, the following equation was fitted to the mean results, using a least squares method on the log-transformed data:

$$
\mathrm{RT}=\beta I^{-\alpha} \text {. }
$$

This function is a simplified form of Piéron's function because the estimation of the third parameter $\left(t_{0}\right)$ would not have been appropriate here. There were two reasons for this; first, we had chosen a small number of luminance levels (five), and second, the highest intensity was still far from the beginning of the asymptotic RT level $\left(40 \mathrm{~cd} / \mathrm{m}^{2}\right.$, according to Mansfield, 1973). The results of each experiment are therefore summarized using two parameters: $-\alpha$ (the exponent of Piéron's function) and CT (characteristic task reaction time), which is the RT computed from the equation fitted to the results for the intermediate level of luminance: $0.81 \mathrm{~cd} / \mathrm{m}^{2}$.

As shown in Figure 3, SRT decreased with increasing luminance. The estimated parameters of Piéron's function $(r=-.98)$ were as follows:

$$
\begin{aligned}
& -\alpha=-0.0558, \\
& \mathrm{CT}=218.67 \mathrm{msec},
\end{aligned}
$$

Mean RT difference between the two extreme levels of luminance was $30 \mathrm{msec}$. A between-subjects analysis of variance (ANOVA) revealed that the intensity effect was significant $[F(4,56)=90.39, p<.001]$. This result was observed for each subject. The individual exponents were as follows: (E.D.) -0.0560 , (D.P.) -0.0536 , (A.C.) -0.0566 , and (A.D.) -0.0568 . The between-subjects standard deviation of mean RT was $24 \mathrm{msec}$. 


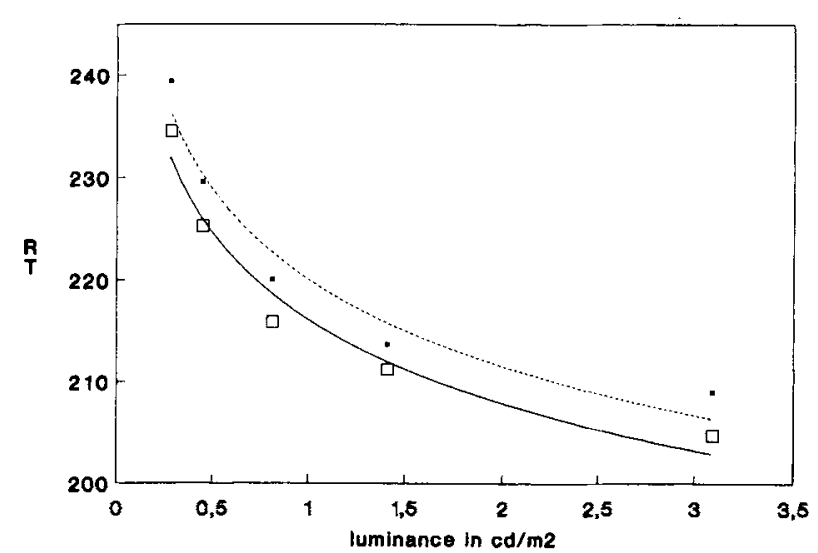

Figure 3. The curves show Piéron's functions fitted to the mean data of the two simple reaction time (SRT) experiments (Experiment 1, solid line; Experiment 2, dotted line). The points (above and below the curves) are the observed mean reaction time (RT) medians (large squares, Experiment 1; small squares, Experiment 2). For both, SRTs decrease with increasing luminance. The curves are strictly parallel.

\section{EXPERIMENT 2 SRT and Orientation}

In this experiment, the spatial uncertainty of the stimuli concerned their orientation $\left( \pm 45^{\circ}\right)$. An orientation discrimination task was presented later (Experiment 5). According to our assumptions, SRT should decrease with increasing luminance of the stimulus, as in Experiment 1, irrespective of the change in the stimulus condition.

\section{Method}

Subjects. Four trained subjects took part in this experiment. One of them was the first author. One of the subjects had not taken part in Experiment 1. All subjects had normal or corrected-tonormal vision.

Procedure. The experimental design was the same as that in Experiment 1. Only the stimuli were different. Two aligned luminous rectangles were presented $\left(20^{\prime} \times 10^{\prime}\right.$ of arc), oriented $\pm 45^{\circ}$ to the vertical. A central gap size of $10^{\prime}$ of arc was chosen to reduce foveal stimulation (Figure 4). The fixation point was in the center of the entire configuration. Because the stimuli were presented more centrally, they were larger than those of Experiment 1 . The choice of their area was made on the basis of equality between the absolute thresholds for the two types of stimuli $\left(0.120 \mathrm{~cd} / \mathrm{m}^{2}\right)$. The task was the same as in Experiment 1 (SRT).

The 4 subjects went through five experimental sessions. Thus 500 RTs were recorded for each level of luminance for each subject.

\section{Results}

The mean difference between the median RTs, according to the orientation of the stimulus, was about $1 \mathrm{msec}$. The data were averaged over the orientation factor.

As shown in Figure 3, SRT decreased with increasing luminance. The estimated parameters of Piéron's function $(r=-.98)$ were as follows:

$$
\begin{aligned}
& -\alpha=-0.0568, \\
& \mathrm{CT}=220.70 \mathrm{msec} .
\end{aligned}
$$

Mean RT difference between the two extreme levels of luminance was $30 \mathrm{msec}$. A between-subjects ANOVA revealed that the intensity effect was significant $[F(4,60)=$ $189.51, p<.001]$. This result held subject by subject with the following exponents: (D.P.) -0.0540 , (A.C.) -0.0581 , (E.D.) -0.0599 , and (B.D.) -0.0557 . The between-subjects standard deviation of mean RT was $14 \mathrm{msec}$.

\section{Conclusion}

The aim of the first two experiments was to estimate the parameters of Piéron's function in SRT tasks for a given range of luminance levels. In these two experiments, RTs decrease similarly with increasing luminance of the stimuli (see Figure 3). In both cases, Piéron's function fit well to the mean results, and the exponents $(-0.0558$ and -0.0568$)$ as well as CT (219 and $223 \mathrm{msec})$ were very similar. Piéron's exponent does not change with the change in stimulus characteristics, at least when the stimuli have the same detection thresholds as was the case here. The interaction between the effect of luminance on RT and the two experimental tasks was not significant $[F(4,56)<1] .{ }^{4}$ Moreover, a similar exponent value was observed in the preliminary experiment $(-0.0513)$ when a large range of luminance levels was used.

\section{Piéron's Law in CRT Tasks with Stimulus-Response Compatibility}

The following experiments were run to verify whether stimulus intensity affects CRT in the same way as it affects SRT. It is to be expected that the CRT will decrease when intensity increases, leading to a similar exponent of Piéron's function despite the increase in CT resulting from the difference in task complexity.

\section{EXPERIMENT 3 CRT and Localization}

In this experiment, the stimulus conditions were identical to those of Experiment 1, but now the subject had to indicate the localization (to the left or to the right of the fixation point) of the target. The spatial location of the response was consistent with the location of the stim-

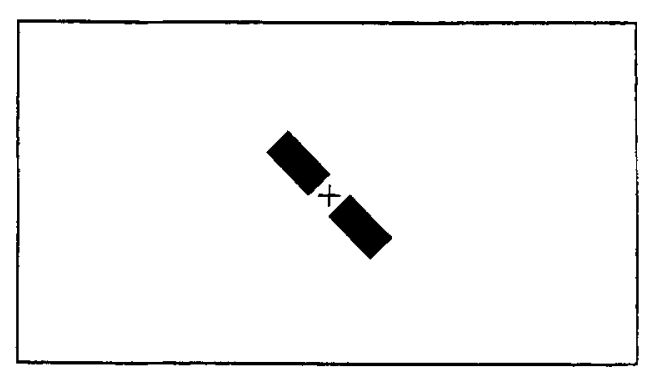

Figure 4. One of the stimuli presented in Experiments 2 and 5, with opposite contrast. Two aligned luminous rectangles were oriented to $-45^{\circ}$, with a central gap. 
ulus (high stimulus-response compatibility; see Luce, 1986).

\section{Method}

Subjects. The same 4 subjects as those in Experiment 1 took part.

Procedure. The stimuli were identical to those used in Experiment 1 . The task was to indicate whether the target appeared to the left (left key) or to the right (right key) of the fixation point. All other conditions corresponded to those of Experiment 1.

\section{Results}

The percentage of correct responses ranged from $94 \%$ to $100 \%$. RT was considered independently from the response type (correct vs. error) in this and the following experiments, since the analysis of the correct responses only led to the same conclusions. The mean difference between the median RTs, according to the localization of the stimulus, was about $1.1 \mathrm{msec}$. For further analyses, the results were averaged over this factor, which had a nonsignificant effect $(F<1)$.

As shown in Figure 5, CRT decreased with increasing luminance of the stimuli. The estimated parameters of Piéron's function $(r=-.98)$ were as follows:

$$
\begin{aligned}
-\alpha & =-0.0547, \\
\mathrm{CT} & =262.79 \mathrm{msec} .
\end{aligned}
$$

Mean RT difference between the two extreme levels of luminance was $35 \mathrm{msec}$. A between-subjects ANOVA revealed that the intensity effect was significant $[F(4,56)=$ $200.29, p<.001]$. This result was observed for each subject. The individual exponents were as follows: (E.D.) -0.0501 , (D.P.) -0.0559 , (A.C.) -0.0574 , and (A.D.) -0.0549 . The between-subjects standard deviation of mean RT was $13 \mathrm{msec}$

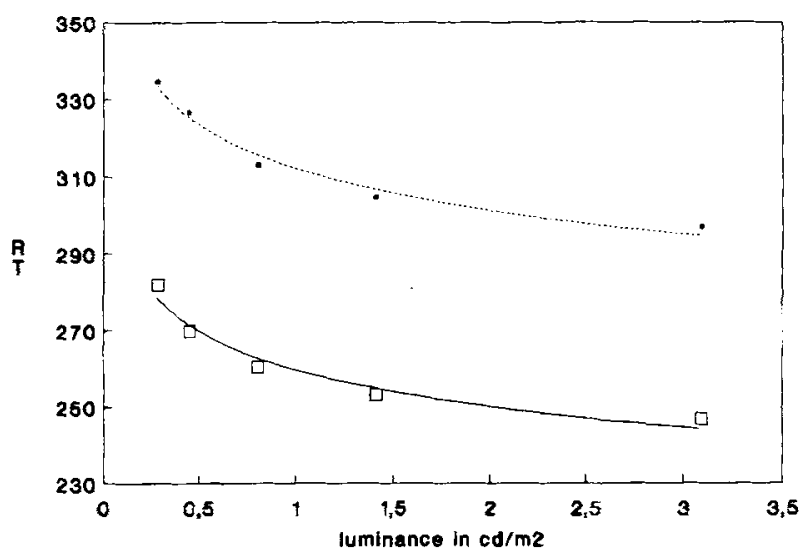

Figure 5. The curves show Piéron's functions fitted to the mean data of the two choice reaction time (CRT) experiments with stimulusresponse compatibility (Experiment 3, solid line; Experiment 4, dotted line). The points (above and below the curves) are the observed mean RT medians (large squares, Experiment 3; small squares, Experiment 4). CRT decreases with increasing luminance. The curves are strictly parallel.

\section{OUTLINE DRAWINGS}
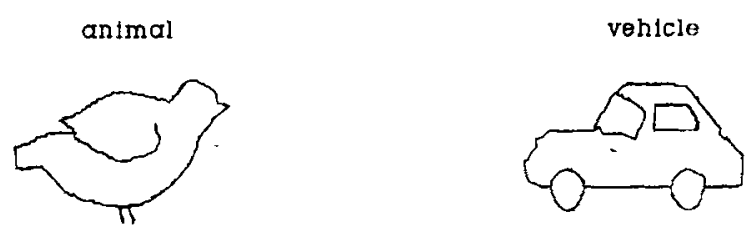

FRAGMENTED FORMS

an ImaI

vehtcle
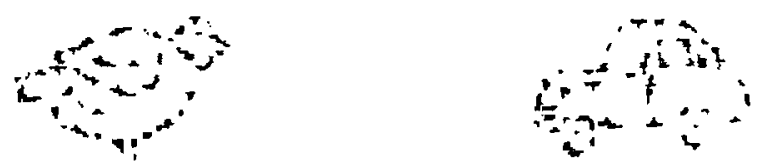

Figure 6. Examples of stimuli presented in Experiment 4, with opposite contrast.

\section{EXPERIMENT 4 CRT and Semantic Categorization}

The aim of the following experiment was to test Piéron's function in a CRT task with a higher level of complexity. A semantic categorization task of drawn objects was chosen. Predictions were the same as those of Experiment 3, though a longer CT was expected because of the higher complexity of the task.

\section{Method}

Subjects. Four trained subjects were run. Two of them were the authors. Two of these subjects had not taken part in the other experiments. All subjects had normal or corrected-to-normal vision.

Procedure. The experimental design was the same as that for Experiment 3. The presentation time of the stimuli was raised to $149 \mathrm{msec}$ (nine frames) in order to obtain a near-perfect categorization performance.

Stimuli were taken from Boucart and Humphreys (1992). Twelve outline drawings of objects $\left(2 \times 2^{\circ}\right.$ or $2 \times 3^{\circ}$ of visual angle) and 12 fragmented forms derived from these outline drawings were presented. Each figure represented an object belonging to one of two semantic categories: vehicle or animal (Figure 6). In order to make sure that the energy content of these stimuli was roughly equivalent, they were composed of identical numbers of light pixels (350 \pm 7 ). Boucart and Bonnet (1990) had in fact found that outline drawings and fragmented forms yield approximately the same detection threshold.

Within a given session, the stimulus type (outline drawing or fragmented form) was constant. In each trial, a pair of stimuli (one vehicle, one animal) was presented at an eccentricity of $1.25^{\circ}$. One appeared to the left and the other to the right of the fixation point. In a given trial, the two stimuli had the same luminance; however, the luminance level changed between trials. The sides of presentation for each semantic category and the luminance levels were randomized over trials.

The subject had to indicate, by pressing the appropriate key, on which side either the animal or the vehicle appeared, depending on the experimental condition. Thus, four experimental conditions were created: (1) outline drawings with the vehicle as the target. (2) outline drawings with the animal as the target, (3) fragmented forms with the vehicle as the target, and (4) fragmented forms with 
the animal as the target. Each experimental session contained 180 trials. Instructions emphasized speed and avoidance of errors.

The subjects went through six sessions for each of the four experimental conditions. Their order was counterbalanced and for each subject, each semantic category, each stimulus type, and each level of luminance, 216 RTs were recorded. Median RTs were computed for each session.

\section{Results}

The mean difference in RT between the two sides on which the target figure was presented was $4 \mathrm{msec}$. The mean difference in RT between the two semantic categories was $0.9 \mathrm{msec}$. For further analyses, the results were averaged over these two factors, which had nonsignificant effects $(F<1)$.

The mean percentage of correct responses was generally high (92.5), but higher for the outline drawings (94.1) than for the fragmented forms (90.9). The subjects had a very similar mean percentage of correct responses (B.D., 89.8; M.B., 93.4; C.B., 92.5; and D.P., 94.3).

As shown in Figure 5, CRT decreased with increasing luminance of the stimuli. The estimated parameters of Piéron's function ( $r=-.94)$ were as follows:

$$
\begin{aligned}
& -\alpha=-0.0518, \\
& \mathrm{CT}=315.84 \mathrm{msec} .
\end{aligned}
$$

Mean RT difference between the two extreme levels of luminance was $39 \mathrm{msec}$. A between-subjects ANOVA revealed that the effect of luminance was significant $[F(4,72)=145.87, p<.001]$. This result held subject by subject with the following exponents: (B.D.) -0.0679 , (M.B.) -0.0549 , (C.B.) -0.0433 , and (D.P.) -0.0424 . The between-subjects standard deviation of mean RT was $26 \mathrm{msec}$.

The effect of stimulus intensity was also significant for each stimulus type: (1) outline drawings $[F(4,72)=$ $96.13, p<.001]$, (2) fragmented forms $[F(4,72)=$ $83.95, p<.001]$. The interaction between stimulus intensity and stimulus type was not significant $[F(4,72)=$ 1.64, n.s.]. The estimated parameters of Piéron's function together with mean RT difference (diff.) between the two extreme levels of luminance are given in Table 1.

Although no systematic difference in the exponents was found between conditions, CTs were, as expected, $50 \mathrm{msec}$ shorter for the outline drawings than for the fragmented forms. The percentage of correct responses decreased by $3 \%$ between the two conditions. Moreover, individual CTs seemed to evolve similarly in the two conditions. The between-subjects standard deviation of mean RT was $24 \mathrm{msec}$ for outline drawings, and $28 \mathrm{msec}$ for fragmented forms.

Because the rate of errors was higher in this experiment, the RTs of errors were tentatively analyzed as a function of stimulus intensity. On average, the RTs of errors tended to decrease with increasing luminance and appeared to cover the same range as the RTs of correct responses. The distribution of error RT had approximately the same mean as that of correct RT.
Table 1

Estimated Parameters of Piéron's Function With Mean Reaction Time Difference Between the Two Extreme Levels of Luminance

\begin{tabular}{lcccc}
\hline \multicolumn{1}{c}{ Stimuli } & $\alpha$ & $r$ & CT & diff. \\
\hline $\begin{array}{l}\text { Outline drawings } \\
\text { Fragmented forms }\end{array}$ & -.0508 & .99 & 290.6 & 35 \\
Note C T, characteristic task reaction time (in milliseconds) & diff., \\
difference.
\end{tabular}

\section{Conclusion}

The mean results of the two CRT experiments can be summarized as follows. Whatever the CRT task (localization or semantic categorization), RT decreased when the luminance of the stimuli increased. In no case did this lead to a change in the exponent of Piéron's function. Comparison of the results of the two experiments reveals that Piéron's law holds for CRT regardless of the complexity of the experimental task. The curves representing the mean results of the two experiments are parallel (Figure 5). Piéron's exponent was similar in the two experiments $(-0.0547$ and -0.0518$)$. The interaction between stimulus intensity and the type of task was not significant $[F(4,56)=1.81$, n.s. $] .{ }^{5}$ (See also note 4.) The increasing complexity of the task only led to an increase in CT. In the semantic categorization task, CT was about $53 \mathrm{msec}$ longer than the CT obtained in the localization task.

The semantic categorization experiment brought further information. Piéron's exponent did not vary with the type of stimulus presented (outline drawing or fragmented form), although the identification of the fragmented forms was more difficult than the identification of the outline drawings $(-0.0508$ and -0.0526$)$. The overall results show that Piéron's exponent is independent of the complexity of the CRT task.

Comparison of the results of SRT and CRT experiments shows that Piéron's law holds for CRT as well as for SRT, whatever the complexity of the experimental task. Moreover, the curves representing the mean results of all four experiments are strictly parallel (Figure 7). Piéron's exponent was similar (Experiment 1: -0.0558 , Experiment 2: -0.0568 , Experiment 3: -0.0547 , Experiment $4:-0.0518)$. The interaction between stimulus intensity and the different tasks (SRT vs. CRT) was not significant $[F(4,56)<1]$. (See notes $4-5$.) The increasing complexity of the task led only to an increase in CT. In CRT tasks, the mean CT was $72 \mathrm{msec}$ longer than in SRT tasks. This result provides further confirmation of the independence of Piéron's exponent and the complexity of the psychophysical task.

\section{Piéron's Law in a CRT Task with No Stimulus-Response Compatibility}

The results of the above experiments suggest that further steps of information processing combine additively to the processing of luminance information. Such additivity is confirmed by the constancy of the exponent of Piéron's function in the different experiments. However, 
in these experiments the perceptual processing of the stimuli was more or less complex. What would happen to the additivity hypothesis if the complexity of the task were defined by the relative difficulty in the choice of the correct response?

A final experiment was run to test whether stimulus intensity affects RT in a CRT experiment with no stimulusresponse compatibility (see, e.g., Biederman \& Kaplan, 1970). It was expected that, provided the observers are trained appropriately, the exponent of Piéron's function would remain the same in this type of task. In fact, our assumption was that further complexity would not alter intensity processing.

\section{EXPERIMENT 5 CRT and Orientation}

In this experiment, the stimulus conditions were identical to those of Experiment 2, but the subject had to indicate the orientation $\left( \pm 45^{\circ}\right)$ of the target. There was no stimulus-response compatibility in this experiment since the spatial location of the response was not consistent with that of the stimulus.

\footnotetext{
Method

Subjects. The same 4 subjects as in Experiment 2 took part in the experiment. One of them was the first author. All subjects had normal or corrected-to-normal vision.

Procedure. The stimulus conditions were identical to those in Experiment 2, but the task was to indicate whether the target was oriented to $-45^{\circ}$ (left key) or to $+45^{\circ}$ (right key) to the vertical. In this experiment, if the subject focused his/her attention to the top of the target, there was some stimulus-response compatibility. If the subject focused his/her attention to the bottom of the target, there was no stimulus-response compatibility, and even an inverse correspondence between stimulus and response location. Since the coding of the response key was arbitrary, the selection of the correct response was more difficult and needed training to become automatic. Two of the subjects had initial difficulty in following the instructions. At the beginning, they completely reversed the responses. The results presented here (five experimental sessions of 500 triais) concern only the range of data for which we could consider that the subjects were trained (low error rate and stable RT over sessions), after about four experimental training sessions of 500 trials.
}

\section{Results}

The percentage of correct responses ranged from $92 \%$ to $100 \%$. The mean difference in RT between the two orientation conditions was $2 \mathrm{msec}$. The results were averaged over this factor, which had a nonsignificant effect $(F<1)$. As shown in Figure 7, CRT decreased with increasing luminance. The estimated parameters of Piéron's function $(r=-.97)$ were as follows:

$$
\begin{aligned}
& -\alpha=-0.0542, \\
& \mathrm{CT}=313.82 \mathrm{msec} .
\end{aligned}
$$

The average RT difference between the two extreme levels of luminance was $40 \mathrm{msec}$. A between-subjects

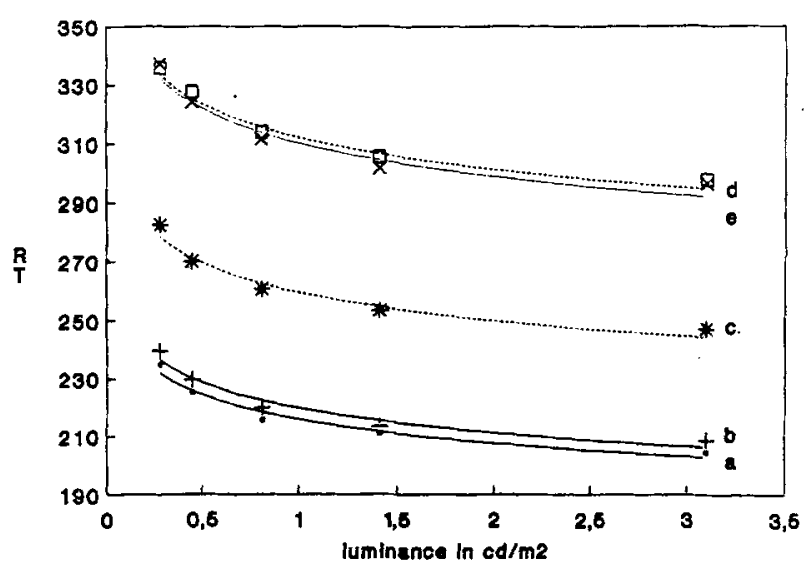

Figure 7. The curves show Piéron's functions fitted to the mean data of the five experiments. All the curves are strictly parallel. (a) SRT localization. (b) SRT orientation. (c) CRT localization. (d) CR'T orientation. (e) Semantic categorization.

ANOVA revealed that the intensity effect was significant $[F(4,60)=199.13, p<.001]$. The effect held for each subject with the following exponents: (D.P.) -0.0508 , (A.C.) -0.0592 , (E.D.) -0.0559 , and (B.D.) -0.0510 . The between-subjects standard deviation of mean RT was $18 \mathrm{msec}$.

\section{Conclusion}

As in the previous experiments, RT decreased with increasing luminance of the stimuli. Piéron's function fit well with the mean results and thus provides a valid estimate for this CRT task. As can be seen in Figure 7, the curve representing the mean results of this experiment is strictly parallel to the curves of all the other data. The exponent is similar to those obtained in all CRT experiments. The interaction between stimulus intensity and the different CRT tasks was not significant $[F(8,112)=$ 1.84, n.s.]. (See notes $4-5$.) The change in complexity of the selection of the correct response did not alter the effect of stimulus intensity.

\section{DISCUSSION}

In these five experiments, we have demonstrated, experiment by experiment, first, that RT decreases with the increase in luminance levels, and second, that this decrease is well described by Piéron's function. Furthermore, the exponent of this function does not seem to be affected by the complexity of the task, at least in the present conditions. Some additional statistical analyses were carried out in order to verify our conclusions. A global analysis over the five experiments revealed that the effect of the task factor was significant $[F(4,56)=95.41$, $p<.001]$ and the luminance factor was also significant $[F(4,56)=220.33, p<.001]$, but the interaction between tasks and intensity was not significant $[F(16,224)=1.65$, n.s.]. (See notes 4-5.) This is a first argument in favor of 
the parallelism of the effects of luminance in the different RT situations used here.

The validity of Piéron's function implies a quadratic regression of the effect of luminance levels on the mean RTs. This was statistically tested in the following way. A linear regression was tested first (Cody \& Smith, 1991), which did not appear to be significant $[F(1,3)=8.69$, n.s.]. If it was, it could not explain more than $74 \%$ of the variance of the averaged data. In a second step, results were transformed to logarithms and a new linear regression analysis was performed on the transformed data. This regression was significant $[F(1,3)=83.31, p<$ .003 ]. It explained $97 \%$ of the variance of the averaged data. The fact that the linear regression on the logtransformed data was significant and explained a larger amount of the variance confirms that the best fit is obtained with the Piéron's hyperbolic function that we have used to describe our data. The function fitted to the averaged data of the five experiments $(r=-.98)$ has the following parameters:

$$
\begin{aligned}
& -\alpha=-0.0543, \\
& \mathrm{CT}=266.71 \mathrm{msec} .
\end{aligned}
$$

The absence of interaction between the intensity and the task, taken together with the results of the above analysis, is consistent with the assumption that the value of the exponent of Piéron's function does not vary from task to task. This conclusion can be supported more directly by comparing the averaged exponent with the five exponents fitted to every experiment. As expected, the Student $t$ test was not significant $[t(4)=0.129$, n.s.].

All these analyses confirm the conclusion that the change in the RTs due to the luminance of the stimuli was about the same in all cases and thus led to statistically parallel functions. This parallelism indicates that the early processing of luminance combines additively with the further and different processings required by each task. The decrease in RT with increase in luminance is explained by the fact that the rate of accumulation of the information, which defines the processing of luminance, decreases when the luminance decreases (e.g., Grice, 1968; Link, 1992).

However, the present results, and in particular the constancy of the exponent of Piéron's function, cannot be obtained if the critical level of that accumulation does not remain constant within and between experiments. Simulations using either Grice's (1968) or Link's (1992) model of accumulation have confirmed that the lower the critical level, the lower is the value of the exponent. Within such models, the constancy of the exponent of Piéron's functions for the same level of luminance necessitates the constancy of the critical level of accumulation of the luminance information.

Clearly, a second condition also has to be met in order to obtain these results: Any processing beyond the luminance processing must be on average of a constant duration within a given experiment. This would not be the case if, for instance, different levels of discrimination were used within an experiment. Hence, constant processing duration is a necessary condition to account for additivity of the two types of processing: one related to luminance, the other to the required binary choice of simple discrimination.

In SRT conditions, the assumed critical level of accumulation is considered as a response criterion (Grice, 1968; Link, 1992). For that reason, it is clearly under the subject's control and it can vary with factors known to modify response criteria. In our experiments, we did not intend to induce such changes. The interpretation of that critical level is rather more complex in the case of CRT. We assumed that it means the end of the luminance processing per se. However, there are reasons to believe that, even as such, it remains under the subject's control. For instance, in Experiment 5, in which there was no stimulusresponse compatibility, this critical level seems to have been initially high, since the exponent of Piéron's function fitted to the data generally declined from session to session during the first four sessions, becoming low and stable after several sessions. At the same time, the mean number of errors declined to a minimum and also became stable.

It should therefore be stressed that these conclusions are valid only if specific methodological requirements are met. Several conditions were chosen in order to ensure that the critical level of accumulation remained low and constant. The first was the use of mixed blocks of luminance levels. The second was the use of trained subjects. Training was considered to be acquired when the RT results became stable within an experiment. In addition, we allowed our subjects to make few errors in order to ensure that their critical level was as low as possible (see, e.g., Luce, 1986). Some changes in the stimulus conditions between experiments were introduced in order to meet these requirements. The area of the stimuli in Experiment 5 was larger than in Experiment 3 in order to make them equally visible as assessed on the basis of their detection thresholds, and confirmed by their nearly equal SRTs. The exposure time used in Experiment 4 was longer than in the other experiments in order to obtain an accuracy of categorization comparable to that of position or orientation discrimination. Regarding the latter, it should be pointed out that the choice of a constant and short exposure time rather than a responseterminated presentation was also intended to ensure a low critical level. In an SRT situation, one of us (Bonnet, Gurlekian, \& Harris, 1992) found an absence of effect of these different conditions with highly trained subjects. However, in some cases, probably when training is not sufficient, response-terminated conditions tend to increase RTs.

In conclusion, the luminance processing and any further processing due to the specific requirements of the psychophysical task combine additively. This is true when the same stimuli are used either for an SRT or a CRT task. The added duration of processing appears to be re- 
lated to the complexity of the task whether it requires deeper processing of the perceptual information or more difficult choice of response, as in the case of a lack of stimulus-response compatibility. However, the constancy of the value of the exponent of Piéron's function strongly argues in favor of a constant duration of initial processing. The results are compatible with a strictly serial model, but also with a cascade model (McClelland, 1979) or a queue-series model (Miller, 1993). However, these issues are beyond the scope of our paper.

\section{REFERENCES}

BARTLETT, J. R., \& DOTY, R. W., SR. (1974). Response of units in striate cortex of squirrel monkeys to visual and electrical stimuli. Journal of Neurophysiology, 37, 621-641.

Biederman, I., \& Kaplan, R. (1970). Stimulus discriminability and S-R compatibility: Evidence for independent effects in choice reaction time. Journal of Experimental Psychology, 86, 434-439.

BONNET, C. (1992a). Psychological scaling within an information processing approach? Behavioral \& Brain Sciences, 15, 560-561.

BONNET, C. (1992b). Sensation and psychophysics of reaction time. International Journal of Psychology, 27, 373.

Bonnet, C., Gurlekian, J., \& Harris, P. (1992). Reaction time and visual area: Searching for the determinants. Bulletin of the Psychonomic Society, 30, 396-398.

BOUCART, M., \& BONNET, C. (1990). Only stimulus energy affects the detectability of visual forms and objects. Bulletin of the Psychonomic Society, 28, 415-417.

BoucarT, M., \& HUMPhREYS, G. W. (1992). Global shape cannot be attended without object identification. Journal of Experimental Psychology: Human Perception \& Performance, 18, 785-806.

BOYNTON, R. M., \& WhITTEN, D. N. (1970). Visual adaptation in monkey cones: Recordings of late receptor potentials. Science, 170, 1423-1426.

Cattell. J. M. (1886). The influence of the intensity of the stimulus on the length of the reaction time. Brain, 8, 512-515.

Cody, R. P., \& SMITH, J. K. (1991). Applied statistics and the SAS programming language. Amsterdam: Elsevier, North-Holland.

EASLAND, G. S., \& Wasserman, G. S. (1979). Multiple intracellular contributions to light adaptation in limulus ommatidia. Vision Research, 19, 1-8.

Everet,, B. L., Hochhaus, L., \& Brown, J. R. (1985). Letter-naming as a function of intensity, degradation, S-R compatibility, and practice. Perception \& Psychophysics, 37, 467-470.

EXNER, S. H. (1868). Über die zu einer Gesichtswahrnehmung nöthige Zeit [On the time necessary to a visual perception]. Sitzungsberichte der Kaiserlicher Akademie der Wissenschaften, 57, 601-632.

GARNER, W. R. (1974). The processing of information and structure. Potomac, MD: Halstead.

Graham, C. H. (1965). Vision and visual perception. New York: Wiley

Grice, G. R. (1968). Stimulus intensity and response evocation. Psychological Review, 75, 359-373.

GRICE, G. R., \& HUNTER, J. J. (1964). Stimulus intensity effects depend upon the type of experimental design. Psychological Review, 71. 247-256.

HEMIL̈̈, S. (1987). The stimulus-response functions of visual systems. Vision Research, 27, 1253-1261.

HINTON, G. E., \& SESNOWSKI, T. J. (1986). Learning and relearning in Boltzmann machines. In D. E. Rumelhart \& I. L. McClelland (Eds.), Parallel distributed processing: Explorations in the microstructure of cognition (pp. 282-317). Cambridge, MA: MIT Press.

LAMING, D. R. J. (1968). Information theory of choice-reaction times London: Academic Press

LaPPIN, J. S., \& DisCH, K. (1972). The latency operating characteristic: Il. Effects of visual stimulus intensity on choice reaction time Journal of Experimental Psychology, 93, 367-372.

LEVICK, W. R. (1973). Variation in the response latency of cat retinal ganglion cells. Vision Research, 13, 837-853.
LiNk, S. W. (1978). The relative judgment theory of the psychometric function. In J. Requin (Ed.), Attention and performance VII (pp. 619-630). Hillsdale, NJ: Erlbaum.

LINK, S. W. (1992). The wave theory of discrimination and similarity. Hillsdale, NJ: Erlbaum

LuCE, R. D. (1986). Response time. New York: Oxford University Press. LuCE, R. D., \& GREEN, D. M. (1972). A neural timing theory for response times and the psychophysics of intensity. Psychological Review, 79, 14-57.

MANSFIELD, R. J. W. (1973). Latency functions in human vision. Vision Research, 13, 2219-2234.

Marrocco, R. T. (1975). Possible neural basis of brightness magnitude estimations. Brain Research, 86, 128-133.

MCCLELland, J. L. (1979). On the time relations of mental processes in cascade. Psychological Review, 86, 287-330.

McGill, W. J. (1961). Loudness and reaction time: A guided tour of the listener's private world. Acta Psychologica, 19, 193-199.

McGill, W. J. (1963). Stochastic latency mechanisms. In R. D. Luce, R. R. Bush, \& E. Galanter (Eds.), Handbook of mathematical psychology (Vol. 1, pp. 309-360). New York: Wiley.

Miller, J. (1993). A queue-series model for reaction time, with discrete-stage and continuous flow models as special cases. Psychological Review, 100, 702-715.

NAKA, K. I., \& Rushton, W. A. (1966). S-potentials from luminosity units in the retina of fish (Cyprinidae). Journal of Physiology, 185, 587-599.

Nissen, M. J. (1977). Stimulus intensity and information processing. Perception \& Psychophysics, 22, 338-352.

Pachella, R. G., \& Fisher, D. F. (1969). Effect of stimulus degradation and similarity on the trade-off between speed and accuracy in absolute judgements. Journal of Experimental Psychology, 81, 7-9.

PiÉron, H. (1914). Recherches sur les lois de variation des temps de latence sensorielle en fonction des intensités excitatrices [On the laws of variation of sensory processing time as a function of the excitatory intensity]. L'Année Psychologique, 20, 17-96.

Piéron, H. (1920). Nouvelles recherches sur l'analyse du temps de latence sensorielle en fonction des intensités excitatrices [Further evidence on the laws of sensory processing time as a function of the excitatory intensity]. L'Année Psychologique, 22, 58-142.

PIÉron, H. (1952). The sensations. New Haven, CT: Yale University Press.

Posner, M. I. (1986). Chronometric explorations of mind. New York: Oxford University Press.

SCHWEICKeRT, R., DAHN, C., \& MCGuigan, K. (1988). Intensity and number of alternatives in hue identification; Piéron's law and choice reaction time. Perception \& Psychophysics, 44, 383-389.

StONE, J., \& FukUdA, Y. (1974). Properties of cat retinal ganglion cells: A comparison of W-cells with X-and Y-cells. Journal of Neurophysiology, 37, 722-748.

TePas, D. I., \& Armington, J. C. (1962). Properties of evoked visual potentials. Vision Research, 2, 449-461.

UENo, T. (1977). Reaction time as a measure of temporal summation at suprathreshold levels. Vision Research, 17, 227-232

Vaughan, H. G., JR., CosTa, L. D., \& Gilden, L. (1966). The functional relation of visual evoked response and reaction time to stimulus intensity. Vision Research, 6, 645-656.

Wundt, W. (1886). Grundzüge der Physiologisches Psychologie [Elements of psychophysics]. Paris: Felix Alcan.

\section{NOTES}

1. Michaelis's function:

$$
a(I)=\frac{I^{p}}{I^{p}+I_{0}^{p}},
$$

where $a$ is the rate at which evidence accrues for a particular alternative, $I$ is the intensity of the stimulus, $I_{0}$ is the threshold intensity, and $p$ is the exponent of the function. 
2. The notion of critical level of accumulation of information will be used here to describe the level of accumulation of information related to intensity necessary before the other processes can start.

3. The notion of response criterion will be used here only to describe the time in which the subject is able to give a response.

4. When analysis of variance was run in order to compare the results observed in Experiment 1 or 3 with results of other experiments, only four experimental sessions were analyzed for 1 subject. In fact, 1 sub- ject (E.D.) ran only through four sessions in Experiments 1 and 3. $F$ was considered significant until $p=.05$.

5. For comparison, the last experimental session (6) of Experiment 4 is not used in this analysis of variance. The other experiments used only five sessions.

(Manuscript received February 8, 1994; revision accepted for publication August 11, 1995.) 